\title{
Non-invasive screening for esophageal varices in patients with liver cirrhosis
}

\author{
Andreas Karatzasa, Christos Konstantakis ${ }^{b}$, loanna Aggeletopoulou', Christina Kalogeropouloud, \\ Konstantinos Thomopoulos', Christos Triantos'
}

Olympion Therapeutirio; St Andreas General Hospital; University Hospital of Patras, Patras, Achaia, Greece

\begin{abstract}
Esophageal varices are one of the main complications of liver cirrhosis. Upper gastrointestinal endoscopy is the gold standard for the detection of esophageal varices. Many less invasive methods for screening of varices have been investigated and the most recent Baveno VI guidelines suggest that endoscopy is not necessary in patients with liver stiffness $<20 \mathrm{kPa}$ and platelets $>150,000 / \mu \mathrm{L}$. A critical review of the literature was performed concerning non-invasive or minimally invasive methods of screening for esophageal varices. Liver and spleen elastography, imaging methods including computed tomography, magnetic resonance imaging and ultrasound, laboratory tests and capsule endoscopy are discussed. The accuracy of each method, and its advantages and limitations compared to endoscopy are analyzed. There are data to support the Baveno VI guidelines, but there is still a lack of large prospective studies and low specificity has been reported for the liver stiffness and platelet count combination. Spleen elastography has shown promising results, as there are data to support its superiority to liver elastography, but it needs further assessment. Computed tomography has shown high diagnostic accuracy and can be part of the diagnostic work up of cirrhotic patients in the future, including screening for varices.
\end{abstract}

Keywords Esophageal varices, elastography, computed tomography, magnetic resonance imaging Ann Gastroenterol 2018; 31 (3): 1-10

\section{Introduction}

Esophageal varices are portosystemic collaterals developed in cirrhotic patients with portal hypertension. Every year, a percentage of patients with cirrhosis (3-12\%) develop esophageal varices and in $8-12 \%$ of patients, progression from small to large varices is detected. Spontaneous regression of small esophageal varices can also be observed, mainly following alcohol abstinence in alcoholic cirrhosis [1,2].

Departments of a Radiology, Olympion Therapeutirio (Andreas Karatzas); 'bastroenterology, St Andreas General Hospital (Christos Konstantakis); ' Gastroenterology, University Hospital of Patras (Ioanna Aggeletopoulou, Konstantinos Thomopoulos, Christos Triantos); ${ }^{\mathrm{d}}$ Radiology, University Hospital of Patras (Christina Kalogeropoulou), Patras, Achaia, Greece

Conflict of Interest: Christos Triantos has received fees as a speaker/ advisory board member and research/travel grants from MSD, Roche, AbbVie, Janssen, Bristol-Myers Squibb, Bayer and Gilead Sciences

Correspondence to: Christos Triantos, MD, D. Stamatopoulou 4, Rio 26504, Patras, Greece, e-mail: chtriantos@hotmail.com

Received 6 September 2017; accepted 26 January 2018; published online 3 March 2018

DOI: https://doi.org/10.20524/aog.2018.0241
In the setting of variceal bleeding a 6-week mortality rate of $11.1-40 \%$ has been reported $[3,4]$. The presence of red spots, the size of varices, and the severity of cirrhosis are considered to be the most important predictors of variceal bleeding [5]. Treatment with $\beta$-blockers can diminish the probability of bleeding by $50 \%$ in patients with medium and large varices [6,7]. At present, the method of choice for identifying the presence and estimating the size of varices is esophagogastroduodenoscopy (EGD). The disadvantages of EGD include the complications associated with endoscopy, especially the need for intravenous sedation [8] and the relatively high cost [9]. These drawbacks have driven the research for new methods of variceal detection. Several minimally or non-invasive methods have been proposed as alternatives to EGD for screening for esophageal varices. The updated Baveno VI guidelines [10] recommend that screening EGD can be avoided in patients with compensated advanced chronic liver disease (cACLD) who have liver stiffness $<20 \mathrm{kPa}$ and a platelet count $>150,000 / \mu \mathrm{L}[10]$.

Non-invasive methods also currently have a distinct role in clinically significant portal hypertension $(\mathrm{CSPH})$ in patients with CACLD [10]. In patients with viral-related CACLD noninvasive methods are sufficient to rule-in CSPH, defining the group of patients at risk of having endoscopic signs of portal hypertension. Liver stiffness measured by transient elastography (TE) $(20-25 \mathrm{kPa})$ can be used alone or in combination with 
platelet count and spleen size [10,11]. Additionally, collateral circulation identified on imaging is sufficient to rule-in CSPH in patients with cACLD of all etiologies. Non-invasive methods do not yet have a well-established role in the follow up of patients with varices.

The purpose of this review is to summarize the accuracy, advantages and disadvantages of non-invasive methods of screening for esophageal varices, focusing on the evidence that exists in the literature as regards avoiding screening by EGD in certain groups of patients.

\section{Materials and methods}

We performed a literature search of PubMed, EMBASE, Web of Science, and CENTRAL in the Cochrane Library from $01 / 2000$ to $01 / 2017$. The key words used were "(fibroscan OR transient elastography OR stiffness OR computed tomography OR magnetic resonance OR platelet spleen ratio OR Doppler OR capsule endoscopy OR ultrasound) AND varices". The search was conducted independently by three reviewers (CT, $\mathrm{AK}$, and $\mathrm{CK}$ ). All manuscripts that compared non-invasive or minimally invasive methods of screening for esophageal varices to EGD were studied. We excluded studies not written in English and studies in children, and included only studies concerning esophageal varices. The references of each full-text article were also reviewed carefully to include studies that met the inclusion criteria. A critical review of the literature was then performed. The studies judged most important, based on the number of patients, methodology, and outcomes, were included in the final review. Special attention was given to the studies validating Baveno VI guidelines. All disagreements were resolved after full discussion within the group of researchers.

The non-invasive methods of screening for esophageal varices investigated are presented in Fig. 1. The accuracy of each of these methods is analyzed in this review. Emphasis is placed on larger-scale studies and meta-analyses. The presence and the cause (if identifiable) of any false-positive or falsenegative results are discussed for each method. Finally, the potential of other methods, apart from liver elastography and platelet count, to serve as a screening method that could replace elastography is elaborated.

\section{Results}

\section{Elastography}

At present, there are three different elastography techniques that measure liver and spleen stiffness. TE (FibroScan) uses a mechanical wave generated by a special transducer, while acoustic radiation force impulse imaging (ARFI) and shear wave elastography (SWE) use sound waves. TE has technical limitations, of which the main ones are ascites, obesity, and narrow intercostal spaces. Although there are new probes that have improved TE in obese patients [12] and patients with

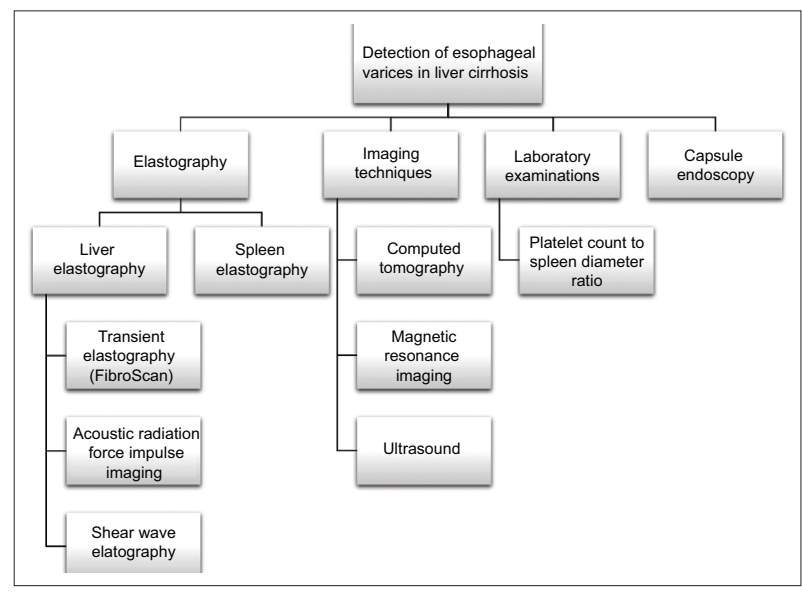

Figure 1 Diagnostic non-invasive methods for the detection of esophageal varices in patients with liver cirrhosis

narrow intercostal spaces, such as children [13], TE remains unfeasible in patients with ascites [14]. ARFI and SWE have partly overcome these limitations and have shown higher success rates [15-19]. The diagnostic accuracy of all three methods is similar, but TE has been validated in more studies. The above methods have been used to correlate liver and spleen stiffness with the presence of varices.

\section{Liver elastography}

Liver stiffness has been proposed as a non-invasive surrogate marker for the presence of esophageal varices and many related studies have been conducted using TE, ARFI or SWE. The reported accuracy in most studies is satisfactory, although it is clear that both liver and spleen elastography are methods that only indirectly estimate the presence of varices, by evaluating the mechanical properties of the liver or spleen, and do not directly appreciate the hemodynamics related to the creation of varices. Nevertheless, a strong correlation between increased liver stiffness and the presence of large varices has been supported.

\section{TE}

A recent meta-analysis [20] that evaluated the accuracy of TE in the prediction of esophageal varices has shown pooled sensitivity and specificity of $84 \%$ and $68 \%$, respectively, for the detection of large varices in patients with liver cirrhosis. The positive and negative likelihood ratios were 2.58 and 0.24 respectively. The results of another meta-analysis were similar, with a pooled sensitivity and specificity of $81 \%$ and $71 \%$, respectively, for the detection of large varices and positive and negative likelihood ratios of 2.63 and 0.27 [21]. The use of liver elastography as a single test in clinical practice remains limited because of the great variety of the cutoff values that have been used for the prediction of the presence of varices, which range from 13.4-29.7 kPa. Additionally, the reported sensitivity and 
specificity of the method varies considerably among studies, ranging from 64 to $96 \%$ and from 44 to $100 \%$, respectively [20-23]. The positive and negative likelihood ratios vary from 1.23 to 26.54 and from 0.05 to 0.75 , respectively. Results are more encouraging for the detection of large varices, with sensitivity varying from 77 to $100 \%$, specificity from 60 to $87 \%$, positive likelihood ratio from 1.65-5.79 and negative likelihood ratio from $0-0.48$. However, the cutoff values that have been used again exhibit considerable variability, ranging between 16.6 and $38.2 \mathrm{kPa}[20,21]$. Applying the cutoff value $(<20 \mathrm{kPa})$ proposed by BAVENO VI to elastography (as a single test) for the detection of large varices in patients with viral hepatitis yielded a sensitivity of approximately $83 \%$ and a negative predictive value of $66.7 \%$, not sufficient to rule out varices [24].

\section{ARFI}

ARFI has also been proven to have high sensitivity and specificity for the detection of varices and the diagnosis of portal hypertension, reaching $84 \%$ and $88 \%$, respectively [25]. High diagnostic accuracy has been reported for the combination of ARFI and spleen diameter/platelet count ratio, with sensitivity and specificity of $90 \%$ and $94.3 \%$ and negative predictive value of $98.3 \%$ for the detection of high risk varices, using a cutoff value of 2.83 [26].

\section{SWE}

There are few studies with measurement of liver stiffness by SWE, but they have shown promising results, with sensitivity and specificity up to $83 \%$ and $66 \%$, respectively [27-29]. Furthermore, a prospective study that directly compared the accuracy of SWE and TE in the detection of varices reported SWE to be superior [15].

The combination of liver stiffness with platelet count increases the accuracy of the method and is incorporated in the BAVENO VI guidelines [10]. BAVENO VI guidelines focus on large varices requiring treatment and report that patients with liver stiffness $<20 \mathrm{kPa}$ and platelet count $>150,000 / \mu \mathrm{L}$ demonstrate a very low risk of having varices that need treatment; thus, they can avoid screening endoscopy. These guidelines have been validated recently by retrospective studies [30-32] and a meta-analysis [33]. Among the studies included in the meta-analysis, only one was prospective [33]. The above studies have shown negative predictive value above 95\% for the combination of liver stiffness measured by elastography and platelet count. Apart from the satisfactory sensitivity, the recent studies confirmed the threshold of $20 \mathrm{kPa}$ for liver stiffness and $150,000 / \mu \mathrm{L}$ for platelet count, with one exception in which a threshold of $25 \mathrm{kPa}$ and $100,000 / \mu \mathrm{L}$ platelets was proposed [34]. Nevertheless, the specificity and positive predictive value of the combination of liver stiffness and platelet count is low (29.6\% and 13.7 respectively) [33].

In other studies, liver stiffness has been combined with spleen size and platelet count and yielded sensitivities and specificities of $70-86.3 \%$ and $86.2-89.6 \%$, respectively $[35,36]$,

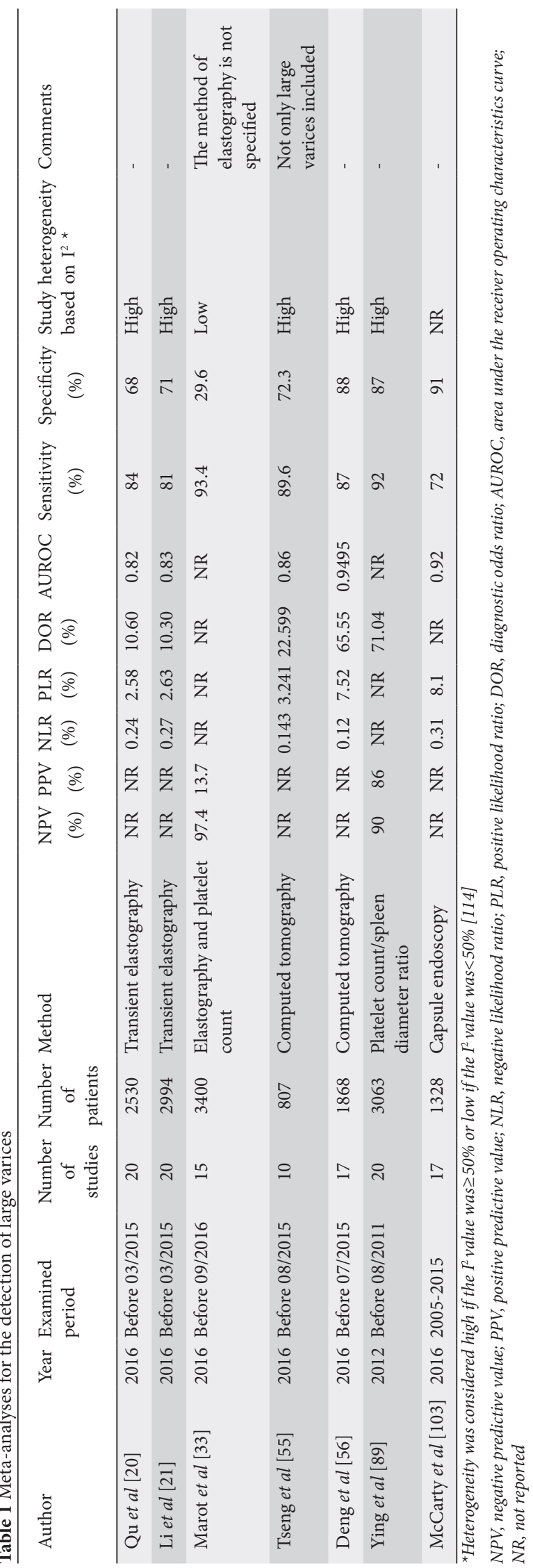


not reaching the sensitivity of the studies combining only liver stiffness with platelet count.

\section{Spleen elastography}

Spleen elastography has emerged lately as a new approach for the detection of esophageal varices, with several studies showing high accuracy [37-42]. Although it is not included in the BAVENO VI guidelines, it has been reported to be superior to liver elastography in several studies that compared both methods in the same group of patients [43-45], with the limitation that it is not an accurate predictor for the presence of varices in alcohol-related cirrhosis [43]. The superiority of spleen elastography compared to liver elastography has been validated in a recent meta-analysis by $\mathrm{Ma}$ et al [46], who reported a pooled sensitivity of $88 \%$ versus $83 \%$ and a pooled specificity of $78 \%$ versus $66 \%$, respectively. The diagnostic odds ratio of spleen stiffness (25.73) was significantly higher than that of liver stiffness (9.54). A high negative predictive value could be achieved with a cutoff value of $18.9 \mathrm{kPa}$, even in a study that showed the method to have only moderate accuracy [47]. When combined with liver elastography it can reach a negative predictive value of $100 \%$, important to define the group of patients that can avoid EGD [48]. Only two studies have reported moderate accuracy of spleen elastography for the detection of varices $[49,50]$.

The technical limitations of liver elastography also apply to spleen elastography. Dedicated devices or software are required, and obesity and ascites may not allow reliable measurements to be obtained. Moreover, because of the small spleen size in some patients, it may be difficult for the operator to obtain good imaging of the splenic parenchyma [51].

In conclusion, spleen elastography appears to be a reliable method with high negative predictive value for the presence of varices. Further evaluation, especially in combination with platelet count and liver elastography, may allow the use of spleen elastography in the future as a screening test, so that EGD can be avoided in patients with negative spleen elastography results.

\section{Imaging techniques}

\section{Computed tomography (CT)}

CT is an imaging modality widely used in cirrhotic patients [52], vastly contributing to the diagnosis and management of the complications of cirrhosis, such as hepatocellular carcinoma (HCC). CT allows direct visualization of esophageal varices after intravenous contrast administration, as serpiginous vessels that protrude in the lumen of the lower esophagus. CT also depicts portosystemic shunts in the abdomen, including periesophageal and perigastric veins $[53,54]$. Although the detection of varices is usually straightforward, it can be challenging to discriminate submucosal from subserosal esophageal varices, and this can be the cause for false-positive and false-negative CT results [53].

CT has been compared to EGD in several studies. Two recent meta-analyses have shown a pooled sensitivity and specificity for the detection of esophageal varices of approximately $89.6 \%$ and $72.3 \%$ [55], and $87 \%$ and $80 \%$ [56], respectively. The sensitivity for the detection of large varices was high in all studies and the pooled sensitivity and specificity for large varices in a meta-analysis by Deng et al was $87 \%$ and $88 \%$, respectively [56]. The positive and negative predictive value for the detection of large varices in the same meta-analysis was $86 \%$ and $90 \%$, respectively.

CT can be a good alternative for the detection of varices in cirrhotic patients with HCC, already undergoing locoregional treatments. The effectiveness of the treatment and the presence of recurrence are evaluated by CT in these patients and Kim et al [43] showed high sensitivity and specificity rates for the detection of esophageal varices ( $91.9 \%$ and $92.2 \%$, respectively) without alteration of the CT protocol.

In the meta-analysis by Tseng et al [55] it was suggested that CT technology (multidetector vs. $<16$ detector CT) may be a reason for the variability among the published results. Nevertheless, the efficacy of standard thick-slice CT, especially for the detection of large varices, was demonstrated by two studies [57,58], without thin slice reconstruction adding accuracy. In addition, like ultrasonography, CT is also an operator-dependent method, but radiologist residents and endoscopists showed similar performance to specialized abdominal radiologists in the detection of large varices. Abdominal radiologists were more efficient in the detection of small varices compared to residents and endoscopists [59].

In the only study directly comparing CT with the platelet/ spleen diameter ratio, CT proved to have higher sensitivity and specificity [53]. This method is better tolerated than endoscopy and more readily accepted by patients, even in studies where air insufflation was used before performing CT [54]. Moreover, CT can be simultaneously used as a screening method for HCC and varices in cirrhotic patients. The main disadvantage of $\mathrm{CT}$ is the radiation dose, although, considering the high mean age of cirrhotic patients, the benefits are likely to outweigh the risk of radiation-induced carcinogenesis [60]. The cost of the method is higher compared to other non-invasive methods, but lower compared to endoscopy [61]. Additionally, intravenous contrast injection is required, which may be contraindicated in patients with impaired renal function or allergy to iodine.

\section{Magnetic resonance imaging (MRI)}

MRI has been evaluated for the detection of esophageal varices, but to a lesser extent compared to CT [62-64]. Direct visualization of esophageal varices by MRI is more challenging than with CT and movement artifacts are much more common. There are reports that detection of varices is feasible [62]. Intravenous injection of gadolinium yielded enhanced results $[63,64]$. The reported accuracy is not high 
(with a sensitivity of less than $81 \%$ ) and in fact appears to be inferior to the accuracy of CT [65].

Magnetic resonance elastography (MRE) has been used to grade the severity of cirrhosis, with the advantage of full organ coverage [66] and low variability for stiffness measurement. Excellent interscan reproducibility [67-69] and inter-reader agreement [70] have been shown. Liver stiffness calculation by MRE with [71] or without [72] contrast-enhanced MRI [71] reached sensitivities of $88.9 \%$ and $96 \%$ and specificities of $56.4 \%$ and $60 \%$, respectively. False-positive results are usually related to the presence of other portosystemic collaterals and false-negative results can occur because of iron deposition in the liver and the presence of large volume ascites [71]. In a study by Morisaka et al [73], MRE was able to discriminate the presence or absence of varices, but failed to distinguish patients with large varices from patients with small varices. Spleen stiffness measured by MRE has been shown to be more strongly correlated with the presence of large varices than with liver stiffness [74,75]. Available data have not demonstrated a clear benefit from the use of MRE compared to ultrasound elastography [71-73]. Additionally, the cost and duration of the examination are higher, and the equipment required is not available in most departments.

The apparent diffusion coefficient (ADC) of the spleen is a parameter that can be calculated simply from a single diffusion weighted sequence on MRI. Recently Razek et al investigated the correlation between the ADC value in the spleen and the presence of varices in cirrhotic patients [76]. There were statistically significant differences among normal volunteers, cirrhotic patients and cirrhotic patients with varices, and when cutoff values of 1.15 and $1.29 \times 10^{-3} \mathrm{~mm}^{2} / \mathrm{sec}$ were used to separate these groups, the resulting areas under the curve were 0.872 and 0.889 , respectively.

MRI has also been used to obtain measurements of liver size, and the ratio between the volume of the right lobe and albumin levels showed a sensitivity of $80 \%$ and a specificity of $83.5 \%$ for the detection of varices [77]. Finally, the presence of Gamna-Gandy bodies (splenic siderotic nodules) in the spleen has been positively correlated with the presence of varices, but the usefulness of this finding needs to be validated [78].

The use of MRI compared to CT has the advantage of the lack of ionizing radiation, but has more contraindications (pacemaker, metallic implants, claustrophobia). Its cost is relatively high, and the measurements of liver stiffness or liver dimensions did not produce better results compared to the studies performed by ultrasound. The correlation of the spleen $\mathrm{ADC}$ with the presence of varices is promising but has to be validated by more studies.

\section{Doppler ultrasonography}

Various indexes measured by Doppler ultrasonography have been proposed as non-invasive predictors of the presence of esophageal varices. In a study that compared several Doppler indexes [79], portal vascular resistance $([0.066 \times$ splenic artery pulsatility index -0.044$] \times$ portal blood flow), hemodynamic liver index (portal vein [PV] diameter/PV mean velocity) and splenoportal index (SPI = spleen long diameter $\times$ spleen short diameter/mean velocity in the PV) were the best predictors for the presence of esophageal varices, with sensitivities of $76 \%, 65 \%$ and $63 \%$ and specificities of $92 \%$, $92 \%$ and $92 \%$, respectively [79]. However, all these parameters failed to accurately predict the presence of large varices. Liver elastography in this study was superior to all the Doppler indexes and had a sensitivity and specificity of $95 \%$ and $100 \%$, respectively, for the detection of varices. SPI has been proved to be a good predictor of the presence of varices in several other studies [80], with a sensitivity up to $96 \%$ [81] but relatively low specificity.

Among the other Doppler indices, the congestion index (CIx) of the PV, first described by Moriyasu [82], has been more widely investigated. This parameter represents the ratio between the cross-sectional area $\left(\mathrm{cm}^{2}\right)$ and the blood flow velocity $(\mathrm{cm} / \mathrm{sec})$ of the PV: CIx $=(\mathrm{pP} 2 / 4) / \mathrm{PV}$ mean, where P is the PV diameter and PV mean represents the mean portal flow velocity [83]. Although the first results were promising for the detection of varices, large varices [84] and red spots [85], other studies failed to confirm these results [86] or demonstrated better predictive values for other indices, such as PV velocity [87].

To date, the role of Doppler ultrasonography remains to be determined and the optimal Doppler index varies among studies. Doppler ultrasonography has the disadvantage of being operator- and equipment-dependent; therefore, the potential for acquiring accurate and reproducible results in relation to the presence of varices is limited.

\section{Contrast-enhanced ultrasound}

In a recent study, contrast enhanced ultrasound was evaluated for the detection of varices [88]. Although direct visualization of esophageal varices was not feasible, the parameters measured yielded high sensitivity and specificity $(96.9 \%$ and $90.0 \%$ respectively) for the detection of varices. Ultrasound has low cost and no ionizing radiation or iodinated contrast material so these promising results have to be validated by further studies [88].

\section{Laboratory tests}

A wide range of laboratory tests have been evaluated as predictors for the presence of varices. The most investigated parameter is the platelet count to spleen diameter ratio [89]. The advantage of this parameter is the fact that a constant cutoff value (909) has been used in most of the studies, which enables its use in clinical practice. A recent metaanalysis, which included 20 studies and 3063 patients [89], showed pooled sensitivities and specificities of $92 \%$ and $87 \%$, respectively, at the threshold of 909 , with a positive predictive value of $86 \%$ and a negative predictive value of $90 \%$. These results have been validated in subgroups of patients with specific etiologies of cirrhosis, including hepatitis C $[35,90]$ and schistosomiasis [91]. However, several studies failed to reproduce the above encouraging results [53,92-96]. 
The spleen volume and the right liver lobe volume, measured by MRI, have also been proposed in combination with platelet count as screening methods for the detection of esophageal varices [97], but the benefit of such a complicated measurement is doubtful, compared to the measurement of the spleen diameter by ultrasound. Additionally, the platelet count alone may predict the presence of large varices, although less accurately, which could be a cheap and readily available screening method in countries with limited resources [98].

In a comparative study of several parameters, the platelet to spleen diameter ratio and the PV diameter measured by CT were the most accurately predictive factors for the presence of large varices in cirrhotic patients with hepatitis C [99]. Various combinations of laboratory findings with demographic parameters and ultrasound parameters [100], serum liver fibrosis markers [101], or even fibrosis parameters from liver biopsy [102], have been shown to correlate with the presence of esophageal varices, but these results have not been validated. A meta-analysis of various non-invasive laboratory findings yielded only moderate accuracy, especially for large varices [99].

In conclusion, although correlations between several laboratory parameters and the presence of varices have been demonstrated, none were strong enough to allow detection of varices with a single laboratory test. As discussed in the section on liver elastography above, the combination of the platelet count with liver stiffness measurements has proven to be a valuable predictor of the presence of varices. Other laboratory measurements need further validation, especially in combination with liver stiffness, and may produce even better results in the future.

\section{Capsule endoscopy}

Wireless video capsule endoscopy was originally designed as a small-bowel imaging device. It was approved by the United States Food and Drug Administration in November 2004 as an alternative technique for the detection of esophageal varices [103]. The diagnostic pooled sensitivity and specificity in a recent meta-analysis of 17 studies [103] were $83 \%$ and $85 \%$, respectively. The pooled sensitivity and specificity for the grading of medium to large varices were $72 \%$ and $91 \%$, respectively. The diagnostic accuracy of this method, especially after taking into consideration its high cost [104], is moderate and not comparable to classic endoscopy.

The use of special strings attached to the capsule, which help control its movement, has yielded the highest accuracy [105]. The sensitivity and specificity in this study reached $96 \%$ and $100 \%$, respectively. However, these results were not validated by another string capsule study, which showed sensitivity and specificity of $84 \%$ and $72 \%$, respectively [106]. Nevertheless, it has been shown that string capsule endoscopy improves visualization of the distal esophagus, which may potentially have an impact on the detection of esophageal varices [107].

A more recent study using the PillCam capsule in 62 patients [108] showed even less satisfactory results, with a sensitivity and specificity of $92 \%$ and $50 \%$ for the detection of varices and $55 \%$ and $91 \%$ for the detection of large varices, respectively. Capsule endoscopy did not detect gastric varices in all 13 patients in the same study [108]. Capsule endoscopy was preferred to EGD by the patients $[108,109]$, although a few patients in some studies had difficulty swallowing it $[105,110,111]$. Capsule endoscopy may have a role in cases of patient refusal or contraindications for EGD and may also improve compliance with endoscopic follow up, but the accuracy of the method remains moderate, despite the advances in the capsule technology. String capsule endoscopy needs to be further evaluated as it holds the potential to yield higher accuracy rates.

\section{Discussion}

The role of non-invasive parameters in the detection of esophageal varices in cirrhotic patients has drawn much attention in recent years. Many studies have been conducted: the most recent meta-analyses are presented in Table 1.

In patients with compensated advanced chronic liver disease, the combination of liver stiffness and platelet count is used to rule out varices, as proposed by BAVENO VI guidelines. This is a great achievement that can reduce the cost and make surveillance for varices less stressful for the patients.

Although the combination of liver stiffness and platelet count, as proposed by the Baveno VI guidelines, is a highly sensitive test for the detection of esophageal varices, it has limited specificity. Further validation with large-scale prospective studies could also be useful. Furthermore, one method or a combination of methods that could reach a negative predictive value of almost $100 \%$ would further increase clinicians' trust in these methods. Promising data were presented in a recent study by Augustin et al [112]; however, more data are needed to validate these results. A test that provided better specificity than the combination of liver stiffness and platelet count would allow EGD to be avoided in larger groups of patients.

The combination of spleen elastography with platelet count appears to be a reasonable choice, considering that spleen elastography has proven to have better accuracy compared to liver elastography in most studies that compared the two methods. If combined with liver elastography and spleen size, its accuracy could be further increased. The new elastography techniques (SWE, ARFI) may help further amplify the diagnostic value of elastography in either liver or spleen.

CT is the only imaging method that allows satisfactory direct visualization of esophageal varices. Although its accuracy in the detection of small varices is suboptimal, it is very precise in the detection of large varices and could be useful in patients not willing to undergo EGD. Stratification of patients according to FibroScan, platelet count and CT could help further increase sensitivity and specificity for the detection of varices; more studies in this direction are needed, considering that CT has not been combined with other tests until now. The ability of CT to detect early HCC could render it a valuable tool in the future for the surveillance of certain groups of cirrhotic patients. It 
is essential to underline the diagnostic capacity of CT for the detection of varices. Radiologists should report on the presence of varices in CT examinations of cirrhotic patients, even when performed for other reasons.

Capsule endoscopy, considering the advances in imaging technology, may serve as an alternative to patients who refuse to undergo a gastroduodenoscopy. However, it is an expensive method, relatively invasive, and its reported accuracy is not yet satisfactory.

Doppler ultrasonography has not shown encouraging results so far in the detection of varices and appears to be less accurate and more time-consuming than elastography. However, a recent study that evaluated the cost-effectiveness and the benefits of two-dimensional ultrasound for the detection of esophageal varices has shown that this technique could be a helpful, simple, and portable tool, saving time and money, and can be used routinely to screen esophageal abnormalities [113].

A recent study evaluated the development of risk prediction models for the detection of varices, based on non-invasive techniques applied in compensated cirrhotic patients [31]. The study showed that the liver stiffness to spleen/platelet score (LSPS) had high discrimination, but none of the non-invasive techniques identified patients with very low risk of all-size varices. However, both LSPS and a model that combined TE and platelet count identified patients with very low risk of varices needing treatment [31].

Emerging techniques that have not yet been adequately evaluated, but whose first results seem to be very promising, are diffusion-weighted imaging on MRI, calculating the ADC value in the spleen, and contrast-enhanced ultrasound. In particular, diffusion-weighted imaging is a single sequence on MRI, fast to perform, and without requiring intravenous contrast. Validation of the first promising results is needed and the combination of this method with other parameters, such as platelet count, needs to be considered.

\section{Concluding remarks}

Non-invasive methods, and specifically elastography, have provided alternatives for the surveillance of certain cirrhotic populations and there is evidence to support the Baveno VI guidelines and to avoid screening EGD in large groups of cirrhotic patients. However, this is an area that could still benefit from further research. Ideally, EGD in the future will be reserved mainly for therapeutic purposes; although this appeared unrealistic a few years ago, advances in technology and more prospective studies could make it feasible.

\section{References}

1. Merli M, Nicolini G, Angeloni S, et al. Incidence and natural history of small esophageal varices in cirrhotic patients. J Hepatol 2003;38:266-272.

2. de Franchis R, Dell'Era A. Non-invasive diagnosis of cirrhosis and the natural history of its complications. Best Pract Res Clin Gastroenterol 2007;21:3-18.

3. Burroughs AK, Triantos CK. Predicting failure to control bleeding and mortality in acute variceal bleeding. J Hepatol 2008;48:185-188.

4. D'Amico G, De Franchis R; Cooperative Study Group. Upper digestive bleeding in cirrhosis. Post-therapeutic outcome and prognostic indicators. Hepatology 2003;38:599-612.

5. Rigo GP, Merighi A, Chahin NJ, et al. A prospective study of the ability of three endoscopic classifications to predict hemorrhage from esophageal varices. Gastrointest Endosc 1992;38:425-429.

6. Tursi T. Use of $\beta$ - blocker therapy to prevent primary bleeding of esophageal varices. J Am Acad Nurse Pract 2010;22:640-647.

7. Thuluvath PJ, Maheshwari A, Jagannath S, Arepally A. A randomized controlled trial of beta-blockers versus endoscopic band ligation for primary prophylaxis: a large sample size is required to show a difference in bleeding rates. Dig Dis Sci 2005;50:407-410.

8. Lichtenstein DR, Jagannath S, Baron TH, et al; Standards of Practice Committee of the American Society for Gastrointestinal Endoscopy. Sedation and anesthesia in GI endoscopy. Gastrointest Endosc 2008;68:815-826.

9. Spiegel BM, Targownik L, Dulai GS, Karsan HA, Gralnek IM. Endoscopic screening for esophageal varices in cirrhosis: Is it ever cost effective? Hepatology 2003;37:366-377.

10. de Franchis R; Baveno VI Faculty. Expanding consensus in portal hypertension: Report of the Baveno VI Consensus Workshop: Stratifying risk and individualizing care for portal hypertension. $J$ Hepatol 2015;63:743-752.

11. Vizzutti F, Arena U, Romanelli RG, et al. Liver stiffness measurement predicts severe portal hypertension in patients with HCV-related cirrhosis. Hepatology 2007;45:1290-1297.

12. Myers RP, Pomier-Layrargues G, Kirsch R, et al. Feasibility and diagnostic performance of the FibroScan XL probe for liver stiffness measurement in overweight and obese patients. Hepatology 2012;55:199-208.

13. Engelmann G, Gebhardt C, Wenning D, et al. Feasibility study and control values of transient elastography in healthy children. Eur J Pediatr 2012;171:353-360.

14. Sandrin L, Fourquet B, Hasquenoph JM, et al. Transient elastography: a new noninvasive method for assessment of hepatic fibrosis. Ultrasound Med Biol 2003;29:1705-1713.

15. Elkrief L, Rautou PE, Ronot M, et al. Prospective comparison of spleen and liver stiffness by using shear-wave and transient elastography for detection of portal hypertension in cirrhosis. Radiology 2015;275:589-598.

16. Ferraioli G, Tinelli C, Zicchetti M, et al. Reproducibility of realtime shear wave elastography in the evaluation of liver elasticity. Eur J Radiol 2012;81:3102-3106.

17. Friedrich-Rust M, Nierhoff J, Lupsor M, et al. Performance of Acoustic Radiation Force Impulse imaging for the staging of liver fibrosis: a pooled meta-analysis. J Viral Hepat 2012;19:e212-e219.

18. Sporea I, Bota S, Jurchis A, et al. Acoustic radiation force impulse and supersonic shear imaging versus transient elastography for liver fibrosis assessment. Ultrasound Med Biol 2013;39:1933-1941.

19. Cassinotto C, Boursier J, de Lédinghen V, et al. Liver stiffness in nonalcoholic fatty liver disease: A comparison of supersonic shear imaging, FibroScan, and ARFI with liver biopsy. Hepatology 2016;63:1817-1827.

20. Qu Y, Li T, Ye Q, Zhang L, Wang L. A beginning or the end? A metaanalysis to assess the diagnostic accuracy of transient elastography for the prediction of esophageal varices. Saudi J Gastroenterol 2016;22:345-352.

21. Li T, Qu Y, Yang B, Xue Y, Wang L. Evaluation of large esophageal varices in cirrhotic patients by transient elastography: a metaanalysis. Rev Esp Enferm Dig 2016;108:464-472.

22. Kazemi F, Kettaneh A, 'N'kontchou G, et al. Liver stiffness 
measurement selects patients with cirrhosis at risk of bearing large oesophageal varices. J Hepatol 2006;45:230-235.

23. Castéra L, Le Bail B, Roudot-Thoraval F, et al. Early detection in routine clinical practice of cirrhosis and oesophageal varices in chronic hepatitis C: comparison of transient elastography (FibroScan) with standard laboratory tests and non-invasive scores. J Hepatol 2009;50:59-68.

24. Al-Hamoudi WK, Abdelrahman AA, Helmy A, et al. The role of FibroScan in predicting the presence of varices in patients with cirrhosis. Eur J Gastroenterol Hepatol 2015;27:1307-1312.

25. Attia D, Schoenemeier B, Rodt T, et al. Evaluation of liver and spleen stiffness with acoustic radiation force impulse quantification elastography for diagnosing clinically significant portal hypertension. Ultraschall Med 2015;36:603-610.

26. Park Y, Kim SU, Park SY, et al. A novel model to predict esophageal varices in patients with compensated cirrhosis using acoustic radiation force impulse elastography. PLoS One 2015;10:e0121009.

27. Grgurević I, Bokun T, Mustapić S, et al. Real-time two-dimensional shear wave ultrasound elastography of the liver is a reliable predictor of clinical outcomes and the presence of esophageal varices in patients with compensated liver cirrhosis. Croat Med J 2015;56:470-481.

28. Kasai Y, Moriyasu F, Saito K, et al. Value of shear wave elastography for predicting hepatocellular carcinoma and esophagogastric varices in patients with chronic liver disease. J Med Ultrason (2001) 2015;42:349-355.

29. Kim TY, Kim TY, Kim Y, Lim S, Jeong WK, Sohn JH. Diagnostic performance of shear wave elastography for predicting esophageal varices in patients with compensated liver cirrhosis. J Ultrasound Med 2016;35:1373-1381.

30. Shibata S, Joshita S, Umemura T, et al. Liver stiffness-spleen sizeto-platelet ratio risk score detects esophageal varices in chronic liver disease. Springerplus 2016;5:998.

31. Abraldes JG, Bureau C, Stefanescu H, et al; Anticipate Investigators. Noninvasive tools and risk of clinically significant portal hypertension and varices in compensated cirrhosis: The "Anticipate" study. Hepatology 2016;64:2173-2184.

32. Maurice JB, Brodkin E, Arnold F, et al. Validation of the Baveno VI criteria to identify low risk cirrhotic patients not requiring endoscopic surveillance for varices. J Hepatol 2016;65:899-905.

33. Marot A, Trepo E, Doerig C, Schoepfer A, Moreno C, Deltenre P. Liver stiffness and platelet count for identifying patients with compensated liver disease at low risk of variceal bleeding. Liver Int 2017;37:707-716.

34. Ding NS, Nguyen T, Iser DM, et al. Liver stiffness plus platelet count can be used to exclude high-risk oesophageal varices. Liver Int 2016;36:240-245.

35. Shibata S, Umemura T, Yamazaki T, et al. Liver stiffness-spleen sizeto-platelet ratio risk score identifies esophageal varices in Japanese patients with chronic hepatitis C. Hepatol Res 2016;46:884-889.

36. Berzigotti A, Seijo S, Arena U, et al. Elastography, spleen size, and platelet count identify portal hypertension in patients with compensated cirrhosis. Gastroenterology 2013;144:102-111.

37. Rizzo L, Attanasio M, Pinzone MR, et al. A new sampling method for spleen stiffness measurement based on quantitative acoustic radiation force impulse elastography for noninvasive assessment of esophageal varices in newly diagnosed HCV-related cirrhosis. Biomed Res Int 2014;2014:365982.

38. Hirooka M, Ochi H, Koizumi Y, et al. Splenic elasticity measured with real-time tissue elastography is a marker of portal hypertension. Radiology 2011;261:960-968.

39. Stefanescu H, Grigorescu M, Lupsor M, Procopet B, Maniu A, Badea R. Spleen stiffness measurement using FibroScan for the noninvasive assessment of esophageal varices in liver cirrhosis patients. J Gastroenterol Hepatol 2011;26:164-170.
40. Sharma P, Kirnake V, Tyagi P, et al. Spleen stiffness in patients with cirrhosis in predicting esophageal varices. Am J Gastroenterol 2013;108:1101-1107.

41. Bota S, Sporea I, Sirli R, et al. Can ARFI elastography predict the presence of significant esophageal varices in newly diagnosed cirrhotic patients? Ann Hepatol 2012;11:519-525.

42. Takuma Y, Nouso K, Morimoto Y, et al. Measurement of spleen stiffness by acoustic radiation force impulse imaging identifies cirrhotic patients with esophageal varices. Gastroenterology 2013; 144:92-101.

43. Kim H, Choi D, Gwak GY, et al. High-risk esophageal varices in patients treated with locoregional therapies for hepatocellular carcinoma: evaluation with regular follow-up liver CT. Dig Dis Sci 2009;54:2247-2252.

44. Takuma Y, Nouso K, Morimoto Y, et al. Portal hypertension in patients with liver cirrhosis: diagnostic accuracy of spleen stiffness. Radiology 2016;279:609-619.

45. Kim HY, Jin EH, Kim W, et al. The role of spleen stiffness in determining the severity and bleeding risk of esophageal varices in cirrhotic patients. Medicine (Baltimore) 2015;94:e1031.

46. Ma X, Wang $\mathrm{L}, \mathrm{Wu} \mathrm{H}$, et al. Spleen stiffness is superior to liver stiffness for predicting esophageal varices in chronic liver disease: a meta-analysis. PLoS One 2016;11:e0165786.

47. Wong GL, Kwok R, Chan HL, et al. Measuring spleen stiffness to predict varices in chronic hepatitis $B$ cirrhotic patients with or without receiving non-selective beta-blockers. J Dig Dis 2016;17:538-546.

48. Buechter M, Kahraman A, Manka P, et al. Spleen and liver stiffness is positively correlated with the risk of esophageal variceal bleeding. Digestion 2016;94:138-144.

49. Vermehren J, Polta A, Zimmermann O, et al. Comparison of acoustic radiation force impulse imaging with transient elastography for the detection of complications in patients with cirrhosis. Liver Int 2012;32:852-858.

50. Calvaruso V, Bronte F, Conte E, Simone F, Craxì A, Di Marco V. Modified spleen stiffness measurement by transient elastography is associated with presence of large oesophageal varices in patients with compensated hepatitis C virus cirrhosis. J Viral Hepat 2013;20:867-874.

51. Giunta M, Conte D, Fraquelli M. Role of spleen elastography in patients with chronic liver diseases. World J Gastroenterol 2016;22:7857-7867.

52. Valls C, Andia E, Roca Y, Cos M, Figueras J. CT in hepatic cirrhosis and chronic hepatitis. Semin Ultrasound CT MR 2002:23:37-61.

53. Karatzas A, Triantos C, Kalafateli M, et al. Multidetector computed tomography versus platelet/spleen diameter ratio as methods for the detection of gastroesophageal varices. Ann Gastroenterol 2016;29:71-78.

54. Kim SH, Kim YJ, Lee JM, et al. Esophageal varices in patients with cirrhosis: multidetector CT esophagography-comparison with endoscopy. Radiology 2007;242:759-768.

55. Tseng YJ, Zeng XQ, Chen J, Li N, Xu PJ, Chen SY. Computed tomography in evaluating gastroesophageal varices in patients with portal hypertension: A meta-analysis. Dig Liver Dis 2016;48:695702.

56. Deng H, Qi X, Guo X. Computed tomography for the diagnosis of varices in liver cirrhosis: a systematic review and meta-analysis of observational studies. Postgrad Med 2017;129:318-328.

57. Yu NC, Margolis D, Hsu M, Raman SS, Lu DS. Detection and grading of esophageal varices on liver CT: comparison of standard and thin-section multiplanar reconstructions in diagnostic accuracy. AJR Am J Roentgenol 2011;197:643-649.

58. Park HS, Kim YJ, Choe WH, et al. Diagnosis of esophageal varices on liver CT: is thin-section reconstruction necessary? Hepatogastroenterology 2015;62:333-340. 
59. Kim H, Choi D, Gwak GY, et al. Evaluation of esophageal varices on liver computed tomography: receiver operating characteristic analyses of the performance of radiologists and endoscopists. J Gastroenterol Hepatol 2009;24:1534-1540.

60. D'Amico G, Garcia-Tsao G, Pagliaro L. Natural history and prognostic indicators of survival in cirrhosis: a systematic review of 118 studies. J Hepatol 2006;44:217-231.

61. Lotfipour AK, Douek M, Shimoga SV, et al. The cost of screening esophageal varices: traditional endoscopy versus computed tomography. J Comput Assist Tomogr 2014;38:963-967.

62. Johnson CD, Ehman RL, Rakela J, Ilstrup DM. MR angiography in portal hypertension: detection of varices and imaging techniques. J Comput Assist Tomogr 1991;15:578-584.

63. Goshima S, Kanematsu M, Kondo H, et al. Detection and grading for esophageal varices in patients with chronic liver damage: comparison of gadolinium-enhanced and unenhanced steady-state coherent MR images. Magn Reson Imaging 2009;27:1230-1235.

64. Matsuo M, Kanematsu M, Kim T, et al. Esophageal varices: diagnosis with gadolinium-enhanced MR imaging of the liver for patients with chronic liver damage. AJR Am J Roentgenol 2003;180:461-466.

65. Lipp MJ, Broder A, Hudesman D, et al. Detection of esophageal varices using CT and MRI. Dig Dis Sci 2011;56:2696-2700.

66. Bensamoun SF, Wang L, Robert L, Charleux F, Latrive JP, Ho Ba Tho MC. Measurement of liver stiffness with two imaging techniques: magnetic resonance elastography and ultrasound elastometry. J Magn Reson Imaging 2008;28:1287-1292.

67. Hines CD, Bley TA, Lindstrom MJ, Reeder SB. Repeatability of magnetic resonance elastography for quantification of hepatic stiffness. J Magn Reson Imaging 2010;31:725-731.

68. Lee DH, Lee JM, Han JK, Choi BI. MR elastography of healthy liver parenchyma: Normal value and reliability of the liver stiffness value measurement. J Magn Reson Imaging 2013;38:1215-1223.

69. Lee Y, Lee JM, Lee JE, et al. MR elastography for noninvasive assessment of hepatic fibrosis: reproducibility of the examination and reproducibility and repeatability of the liver stiffness value measurement. J Magn Reson Imaging 2014;39:326-331.

70. Runge JH, Bohte AE, Verheij J, et al. Comparison of interobserver agreement of magnetic resonance elastography with histopathological staging of liver fibrosis. Abdom Imaging 2014;39:283-290.

71. Shin SU, Lee JM, Yu MH, et al. Prediction of esophageal varices in patients with cirrhosis: usefulness of three-dimensional MR elastography with echo-planar imaging technique. Radiology 2014;272:143-153.

72. Sun HY, Lee JM, Han JK, Choi BI. Usefulness of MR elastography for predicting esophageal varices in cirrhotic patients. J Magn Reson Imaging 2014;39:559-566.

73. Morisaka H, Motosugi U, Ichikawa $\mathrm{T}$, et al. MR-based measurements of portal vein flow and liver stiffness for predicting gastroesophageal varices. Magn Reson Med Sci 2013;12:77-86.

74. Morisaka H, Motosugi U, Ichikawa S, Sano K, Ichikawa T, Enomoto N. Association of splenic MR elastographic findings with gastroesophageal varices in patients with chronic liver disease. J Magn Reson Imaging 2015;41:117-124.

75. Ronot M, Lambert S, Elkrief L, et al. Assessment of portal hypertension and high-risk oesophageal varices with liver and spleen three-dimensional multifrequency MR elastography in liver cirrhosis. Eur Radiol 2014;24:1394-1402.

76. Razek AA, Massoud SM, Azziz MR, El-Bendary MM, Zalata K, Motawea EM. Prediction of esophageal varices in cirrhotic patients with apparent diffusion coefficient of the spleen. Abdom Imaging 2015;40:1465-1469.

77. Li H, Chen TW, Li ZL, et al. Albumin and magnetic resonance imaging-liver volume to identify hepatitis B-related cirrhosis and esophageal varices. World J Gastroenterol 2015;21:988-996.

78. Zhang J, Tao R, You Z, et al. Gamna-Gandy bodies of the spleen detected with susceptibility weighted imaging: maybe a new potential non-invasive marker of esophageal varices. PLoS One 2013;8:e55626.

79. Bintintan A, Chira RI, Bintintan VV, et al. Value of hepatic elastography and Doppler indexes for predictions of esophageal varices in liver cirrhosis. Med Ultrason 2015;17:5-11.

80. Mahmoud HS, Mostafa EF, Mohammed MA. Role of portal haemodynamic parameters in prediction of oesophageal varices in cirrhotic patients. Arab J Gastroenterol 2014;15:130-134.

81. Chakrabarti R, Sen D, Khanna V. Is non-invasive diagnosis of esophageal varices in patients with compensated hepatic cirrhosis possible by duplex Doppler ultrasonography? Indian J Gastroenterol 2016;35:60-66

82. Moriyasu F, Nishida O, Ban N, et al. "Congestion index" of the portal vein. AJR Am J Roentgenol 1986:146:735-739.

83. Binţinţan A, Chira RI, Mircea PA. Non-invasive ultrasound-based diagnosis and staging of esophageal varices in liver cirrhosis. A systematic review of the literature published in the third millenium. Med Ultrason 2013;15:116-124.

84. Grace ND, Groszmann RJ, Garcia-Tsao G, et al. Portal hypertension and variceal bleeding: an AASLD single topic symposium. Hepatology 1998:28:868-880.

85. Plestina S, Pulanić R, Kralik M, Plestina S, Samarzija M. Color Doppler ultrasonography is reliable in assessing the risk of esophageal variceal bleeding in patients with liver cirrhosis. Wien Klin Wochenschr 2005;117:711-717.

86. Colli A, Fraquelli M, Pometta R, Cocciolo M, Visentin S, Conte D. Renovascular impedance and esophageal varices in patients with Child-Pugh class A cirrhosis. Radiology 2001:219:712-715.

87. Shastri M, Kulkarni S, Patell R, Jasdanwala S. Portal vein Doppler: a tool for non-invasive prediction of esophageal varices in cirrhosis. J Clin Diagn Res 2014;8:MC12-MC15.

88. Qiu L, Zhang X, Liu D, Qian L, Hu X. Contrast-enhanced ultrasonography diagnostic evaluation of esophageal varices in patients with cirrhosis. Ultrasound Q 2016;32:136-143.

89. Ying L, Lin X, Xie ZL, Hu YP, Shi KQ. Performance of platelet count/spleen diameter ratio for diagnosis of esophageal varices in cirrhosis: a meta-analysis. Dig Dis Sci 2012;57:1672-1681.

90. Agha A, Anwar E, Bashir K, Savarino V, Giannini EG. External validation of the platelet count/spleen diameter ratio for the diagnosis of esophageal varices in hepatitis C virus-related cirrhosis. Dig Dis Sci 2009;54:654-660.

91. Xu XD, Xu CF, Dai JJ, Qian JQ, Pin X. Ratio of platelet count/spleen diameter predicted the presence of esophageal varices in patients with schistosomiasis liver cirrhosis. Eur J Gastroenterol Hepatol 2016;28:588-591.

92. Cammà C, Petta S, Di Marco V, et al. Insulin resistance is a risk factor for esophageal varices in hepatitis $\mathrm{C}$ virus cirrhosis. Hepatology 2009;49:195-203.

93. Hong WD, Zhu QH, Huang ZM, et al. Predictors of esophageal varices in patients with HBV-related cirrhosis: a retrospective study. BMC Gastroenterol 2009;9:11.

94. Schwarzenberger E, Meyer T, Golla V, Sahdala NP, Min AD. Utilization of platelet count spleen diameter ratio in predicting the presence of esophageal varices in patients with cirrhosis. J Clin Gastroenterol 2010;44:146-150.

95. Barrera F, Riquelme A, Soza A, et al. Platelet count/spleen diameter ratio for non-invasive prediction of high risk esophageal varices in cirrhotic patients. Ann Hepatol 2009;8:325-330.

96. Sarangapani A, Shanmugam C, Kalyanasundaram M, Rangachari B, Thangavelu P, Subbarayan JK. Noninvasive prediction of large esophageal varices in chronic liver disease patients. Saudi J Gastroenterol 2010;16:38-42. 
97. Chen XL, Chen TW, Zhang XM, et al. Platelet count combined with right liver volume and spleen volume measured by magnetic resonance imaging for identifying cirrhosis and esophageal varices. World J Gastroenterol 2015;21:10184-10191.

98. Abd-Elsalam S, Habba E, Elkhalawany W, et al. Correlation of platelets count with endoscopic findings in a cohort of Egyptian patients with liver cirrhosis. Medicine (Baltimore) 2016;95:e3853.

99. Deng H, Qi X, Guo X. Diagnostic accuracy of APRI, AAR, FIB4, FI, king, lok, forns, and FibroIndex scores in predicting the presence of esophageal varices in liver cirrhosis: a systematic review and meta-analysis. Medicine (Baltimore) 2015;94:e1795.

100. Cho EJ, Kim MY, Lee JH, et al. Diagnostic and prognostic values of noninvasive predictors of portal hypertension in patients with alcoholic cirrhosis. PLoS One 2015;10:e0133935.

101. Qi X, Li H, Chen J, et al. Serum liver fibrosis markers for predicting the presence of gastroesophageal varices in liver cirrhosis: a retrospective cross-sectional study. Gastroenterol Res Pract 2015;2015:274534.

102. Wu QM, Zhao XY, You H. Quantitative fibrosis parameters highly predict esophageal-gastro varices in primary biliary cirrhosis. Eur Rev Med Pharmacol Sci 2016;20:1037-1043.

103. McCarty TR, Afinogenova Y, Njei B. Use of wireless capsule endoscopy for the diagnosis and grading of esophageal varices in patients with portal hypertension: a systematic review and metaanalysis. J Clin Gastroenterol 2017;51:174-182.

104. Spiegel BM, Esrailian E, Eisen G. The budget impact of endoscopic screening for esophageal varices in cirrhosis. Gastrointest Endosc 2007;66:679-692.

105. Ramirez FC, Hakim S, Tharalson EM, Shaukat MS, Akins R. Feasibility and safety of string wireless capsule endoscopy in the diagnosis of esophageal varices. Am J Gastroenterol 2005;100:1065-1071.
106. Stipho S, Tharalson E, Hakim S, Akins R, Shaukat M, Ramirez FC. String capsule endoscopy for screening and surveillance of esophageal varices in patients with cirrhosis. J Interv Gastroenterol 2012;2:54-60.

107. Chen WS, Zhu LH, Li DZ, Chen L, Wu YL, Wang W. String esophageal capsule endoscopy with real-time viewing improves visualization of the distal esophageal Z-line: a prospective, comparative study. Eur J Gastroenterol Hepatol 2014;26:309-312.

108. Krok KL, Wagennar RR, Kantsevoy SV, Thuluvath PJ. Esophageal capsule endoscopy is not the optimal technique to determine the need for primary prophylaxis in patients with cirrhosis. Arch Med Sci 2016;12:365-371.

109. Sacher-Huvelin S, Calès P, Bureau C, et al. Screening of esophageal varices by esophageal capsule endoscopy: results of a French multicenter prospective study. Endoscopy 2015;47:486-492.

110. Lapalus MG, Dumortier J, Fumex F, et al. Esophageal capsule endoscopy versus esophagogastroduodenoscopy for evaluating portal hypertension: a prospective comparative study of performance and tolerance. Endoscopy 2006;38:36-41.

111. Frenette CT, Kuldau JG, Hillebrand DJ, Lane J, Pockros PJ. Comparison of esophageal capsule eSndoscopy and esophagogastroduodenoscopy for diagnosis of esophageal varices. World J Gastroenterol 2008;14:4480-4485.

112. Augustin S, Pons M, Maurice JB, et al. Expanding the Baveno VI criteria for the screening of varices in patients with compensated advanced chronic liver disease. Hepatology 2017;66:1980-1988.

113. Abd Elrazek AE, Eid KA, El-Sherif AE, Abd El Al UM, ElSherbiny SM, Bilasy SE. Screening esophagus during routine ultrasound: medical and cost benefits. Eur J Gastroenterol Hepatol 2015;27:8-12.

114. Whiting PF, Rutjes AW, Westwood ME, et al; QUADAS-2 Group. QUADAS-2: a revised tool for the quality assessment of diagnostic accuracy studies. Ann Intern Med 2011;155:529-536. 